TAIWANESE JOURNAL OF MATHEMATICS

Vol. 8, No. 3, pp. 489-497, September 2004

This paper is available online at http://www.math.nthu.edu.tw/tjm/

\title{
ON THE PÓLYA-SCHIFFER CONVEXITY THEOREM AND ITS APPLICATIONS FOR EIGENVALUES OF VIBRATING STRINGS
}

\author{
Min-Jei Huang
}

\begin{abstract}
We consider eigenvalue problems for the vibrating string

$$
u^{\prime \prime}(x)+\lambda \rho(x) u(x)=0, \quad u(0)=u(a)=0
$$

where the density $\rho(x)$ is a positive continuous function on $[0, a]$. Let $\lambda_{n}(t)$ be the $n$th eigenvalue of the string with $\rho=\rho(x, t)$. A classical convexity theorem of Polya and Schiffer states that for any $k \geq 1$, the $\operatorname{sum} \sum_{n=1}^{k} \frac{1}{\lambda_{n}(t)}$ is a convex function of $t$ if $\rho(x, t)$ is convex with respect to $t$. In this paper, we shall give a different approach to this result based on variational analysis. The ideas used also lead to applications in the case of symmetric densities and in the case of concave densities.
\end{abstract}

\section{INTRODUCTION}

If a string has a density given by a positive continuous function $\rho(x)$ defined on the interval $[0, a]$ and is fixed at its end points under unit tension, then the natural frequencies of vibration of the string are determined by the eigenvalues of the boundary value problem

$$
u^{\prime \prime}(x)+\lambda \rho(x) u(x)=0, \quad u(0)=u(a)=0 .
$$

Let $L_{\rho}^{2}([0, a])$ denote the Hilbert space which consists of those complex-valued measurable functions $f$ such that

$$
\int_{0}^{a}|f(x)|^{2} \rho(x) d x<\infty .
$$

The inner-product in $L_{\rho}^{2}([0, a])$ is defined by

Received November 7, 2002; revised June 30, 2003.

Communicated by Sze-Bi Hsu.

2000 Mathematics Subject Classification: 34L15.

Key words and phrases: Eigenvalue, Eigenfunction, Convexity theorem, Concave density, Symmetric density. 


$$
\langle f, g\rangle=\int_{0}^{a} f(x) \overline{g(x)} \rho(x) d x .
$$

As is well-known, the eigenvalues of (1) form a strictly increasing sequence of positive numbers which depend on the density $\rho(x)$. We denote them accordingly by

$$
0<\lambda_{1}[\rho]<\lambda_{2}[\rho]<\lambda_{3}[\rho]<\cdots .
$$

Also, the corresponding normalized eigenfunctions $u_{1}(x), u_{2}(x), u_{3}(x), \cdots$ can be chosen so as to be real-valued and to form a complete orthonormal basis for $L_{\rho}^{2}([0, a])$.

Suppose that $\rho(\cdot, t)$ is a one-parameter family of densities, and let $\lambda_{n}(t)$ be the $n$th eigenvalue of (1) with $\rho=\rho(x, t)$. It is a classical result that for any $k \geq 1$, the sum $\sum_{n=1}^{k} \frac{1}{\lambda_{n}(t)}$ is a convex function of $t$ if $\rho(x, t)$ is convex with respect to $t$ (Polya and Schiffer [4]). This convexity result was established in [4] by using Poincaré's theorem and the method of transplanting extremal functions. In the present paper, we shall give a different approach to this result based on variational analysis. The ideas used also lead to applications in the case of symmetric densities and in the case of concave densities.

In order to apply the idea of perturbation theory to eigenvalues, we assume that $\frac{\partial \rho}{\partial t}(x, t)$ exists. Then there is a simple formula for the derivative of $\lambda_{n}(t)$ :

$$
\frac{d}{d t} \lambda_{n}(t)=-\lambda_{n}(t) \int_{0}^{a} \frac{\partial \rho}{\partial t}(x, t) u_{n}^{2}(x, t) d x,
$$

where $u_{n}(x, t)$ is the normalized eigenfunction corresponding to the eigenvalue $\lambda_{n}(t)$ (see Huang [1] or Keller [3]). The derivatives of higher order can be derived if we increase the differentiability conditions on $\rho(x, t)$. Here we note the following basic formula that we need.

Theorem 1. (the second-order perturbation formula)

$$
\begin{aligned}
\frac{d^{2}}{d t^{2}} \lambda_{n}(t)= & 2 \lambda_{n}(t)\left[\int_{0}^{a} \frac{\partial \rho}{\partial t}(x, t) u_{n}^{2}(x, t) d x\right]^{2}-\lambda_{n}(t) \int_{0}^{a} \frac{\partial^{2} \rho}{\partial t^{2}}(x, t) u_{n}^{2}(x, t) d x \\
& +2 \lambda_{n}^{2}(t) \sum_{j \neq n} \frac{1}{\lambda_{n}(t)-\lambda_{j}(t)}\left[\int_{0}^{a} \frac{\partial \rho}{\partial t}(x, t) u_{n}(x, t) u_{j}(x, t) d x\right]^{2} .
\end{aligned}
$$

Proof. See the Appendix in Section 3.

As a consequence of Theorem 1, we obtain the following convexity result. 
Theorem 2. If $\frac{\partial^{2} \rho}{\partial t^{2}}(x, t) \geq 0$, then, for any $k \geq 1$,

$$
\frac{d^{2}}{d t^{2}}\left[\sum_{n=1}^{k} \frac{1}{\lambda_{n}(t)}\right] \geq 0
$$

Proof. Since $\frac{\partial^{2} \rho}{\partial t^{2}}(x, t) \geq 0$, we have from Theorem 1 that

$$
\lambda_{n}^{\prime \prime}(t) \leq 2 \lambda_{n}(t) A_{n, n}^{2}(t)+2 \lambda_{n}^{2}(t) \sum_{j \neq n} \frac{1}{\lambda_{n}(t)-\lambda_{j}(t)} A_{n, j}^{2}(t)
$$

where $A_{n, j}(t)=\int_{0}^{a} \frac{\partial \rho}{\partial t}(x, t) u_{n}(x, t) u_{j}(x, t) d x$. So,

$$
\frac{\lambda_{n}^{\prime \prime}(t)-2 \lambda_{n}(t) A_{n, n}^{2}(t)}{2 \lambda_{n}^{2}(t)} \leq \sum_{j \neq n} \frac{1}{\lambda_{n}(t)-\lambda_{j}(t)} A_{n, j}^{2}(t) .
$$

It follows that

$$
\begin{aligned}
{\left[\sum_{n=1}^{k} \frac{1}{\lambda_{n}(t)}\right]^{\prime \prime} } & =\sum_{n=1}^{k} \frac{2\left[\lambda_{n}^{\prime}(t)\right]^{2}-\lambda_{n}(t) \lambda_{n}^{\prime \prime}(t)}{\lambda_{n}^{3}(t)} \\
& =\sum_{n=1}^{k} \frac{2 \lambda_{n}(t) A_{n, n}^{2}(t)-\lambda_{n}^{\prime \prime}(t)}{\lambda_{n}^{2}(t)} \\
& \geq \sum_{n=1}^{k} \sum_{j \neq n} \frac{-2}{\lambda_{n}(t)-\lambda_{j}(t)} A_{n, j}^{2}(t) \\
& =\sum_{n=1}^{k} \sum_{j=k+1}^{\infty} \frac{-2}{\lambda_{n}(t)-\lambda_{j}(t)} A_{n, j}^{2}(t) \\
& \geq 0
\end{aligned}
$$

where we have used (2) in the second step and the fact that $A_{n, j}(t)=A_{j, n}(t)$ in the fourth step.

\section{Applications}

Let $\rho(x)$ be a density on $[0, a]$. We define the density $\rho_{s}(x)$ by

$$
\rho_{s}(x)=\frac{1}{2}[\rho(x)+\rho(a-x)], \quad 0 \leq x \leq a .
$$

It is clear that $\rho_{s}(x)$ is symmetric about $x=a / 2$ and

$$
\int_{0}^{a} \rho_{s}(x) d x=\int_{0}^{a} \rho(x) d x .
$$


To compare the eigenvalues $\lambda_{n}[\rho]$ with $\lambda_{n}\left[\rho_{s}\right]$, let us put $c=\max _{x \in[0, a]} \frac{\rho(a-x)}{\rho(x)}$. Then $\frac{1}{c} \rho(x) \leq \rho(a-x) \leq c \rho(x)$ so that

$$
\frac{c+1}{2 c} \rho(x) \leq \rho_{s}(x) \leq \frac{c+1}{2} \rho(x) .
$$

It follows that

$$
\frac{2 c}{c+1} \lambda_{n}[\rho]=\lambda_{n}\left[\frac{c+1}{2 c} \rho\right] \geq \lambda_{n}\left[\rho_{s}\right] \geq \lambda_{n}\left[\frac{c+1}{2} \rho\right]=\frac{2}{c+1} \lambda_{n}[\rho] .
$$

From this we obtain, in particular, that for any $k \geq 1$,

$$
\frac{c+1}{2} \sum_{n=1}^{k} \frac{1}{\lambda_{n}[\rho]} \geq \sum_{n=1}^{k} \frac{1}{\lambda_{n}\left[\rho_{s}\right]}
$$

An improvement on this inequality is given in the next theorem.

Theorem 3. For any $k \geq 1$,

$$
\sum_{n=1}^{k} \frac{1}{\lambda_{n}[\rho]} \geq \sum_{n=1}^{k} \frac{1}{\lambda_{n}\left[\rho_{s}\right]}
$$

where the equality holds only when $\rho=\rho_{s}$; i.e., only when $\rho$ is symmetric about $x=a / 2$.

Proof. We consider the one-parameter family of densities: $\rho(x, t)=t \rho(x)+$ $(1-t) \rho_{s}(x)$, where $0 \leq t \leq 1$. Fix $k$ and let $\Lambda(t)=\sum_{n=1}^{k} \frac{1}{\lambda_{n}(t)}$. Since $\frac{\partial^{2} \rho}{\partial t^{2}}(x, t)=$ 0 , we have by Theorem 2 that $\Lambda^{\prime \prime}(t) \geq 0$. This implies that $\Lambda(t)-\Lambda(0) \geq \Lambda^{\prime}(0) t$ for $0 \leq t \leq 1$. In particular, taking $t=1$, we get

$$
\sum_{n=1}^{k} \frac{1}{\lambda_{n}[\rho]}-\sum_{n=1}^{k} \frac{1}{\lambda_{n}\left[\rho_{s}\right]}=\Lambda(1)-\Lambda(0) \geq \Lambda^{\prime}(0) .
$$

Next, by (2), we have

$$
\left.\left[\frac{1}{\lambda_{n}(t)}\right]^{\prime}\right|_{t=0}=\frac{-\lambda_{n}^{\prime}(0)}{\lambda_{n}^{2}(0)}=\frac{1}{\lambda_{n}(0)} \int_{0}^{a}\left[\rho(x)-\rho_{s}(x)\right] u_{n}^{2}(x, 0) d x .
$$

Since the density $\rho_{s}(x)$ is symmetric about $x=a / 2$, the corresponding normalized eigenfunctions $u_{n}(x, 0)$ satisfy $u_{n}^{2}(a-x, 0)=u_{n}^{2}(x, 0)$ for all $n=1,2, \cdots$. On the other hand, the function $\rho(x)-\rho_{s}(x)$ is antisymmetric about $x=a / 2$. It follows 
that $\left.\left[\frac{1}{\lambda_{n}(t)}\right]^{\prime}\right|_{t=0}=0$, and hence that $\Lambda^{\prime}(0)=0$. This together with (5) proves the desired inequality.

Finally, if the equality holds in (4), then $\Lambda(1)=\Lambda(0)$. Since $\Lambda^{\prime \prime}(t) \geq 0$ and $\Lambda^{\prime}(0)=0$, this implies that $\Lambda(t)$ is constant for $0 \leq t \leq 1$, and so $\Lambda^{\prime \prime}(t)=0$. On using (3) and taking $t=0$, we find that

$$
A_{n, j}(0)=\int_{0}^{a}\left[\rho(x)-\rho_{s}(x)\right] u_{n}(x, 0) u_{j}(x, 0) d x=0
$$

for $1 \leq n \leq k$ and $j \geq k+1$. Thus, for $1 \leq n \leq k$, we have

$$
\begin{aligned}
\frac{\rho(x)-\rho_{s}(x)}{\rho_{s}(x)} u_{n}(x, 0) & =\sum_{j=1}^{\infty}\left\{\int_{0}^{a} \frac{\rho(x)-\rho_{s}(x)}{\rho_{s}(x)} u_{n}(x, 0) u_{j}(x, 0) \rho_{s}(x) d x\right\} u_{j}(x, 0) \\
& =\sum_{j=1}^{\infty} A_{n, j}(0) u_{j}(x, 0) \\
& =\sum_{j=1}^{k} A_{n, j}(0) u_{j}(x, 0) .
\end{aligned}
$$

This shows that for each $x \in(0, a), \frac{\rho(x)-\rho_{s}(x)}{\rho_{s}(x)}$ is an eigenvalue of the $k \times k$ matrix $\left[A_{n, j}(0)\right]_{1 \leq n, j \leq k}$. Since $\frac{\rho-\rho_{s}}{\rho_{s}}$ is a continuous function on $[0, a]$, it follows that $\frac{\rho-\rho_{s}}{\rho_{s}}$ must be constant. Hence $\rho=\rho_{s}$, because $\rho\left(\frac{a}{2}\right)=\rho_{s}\left(\frac{a}{2}\right)$. This completes the proof of the theorem.

For the constant density $\rho(x)=1$, the eigenvalues and eigenfunctions are wellknown. We have

$$
\lambda_{n}[1]=n^{2} \pi^{2} / a^{2}, \quad n=1,2, \cdots ;
$$

and the corresponding normalized eigenfunctions can, for example, be taken as

$$
u_{n}(x)=\sqrt{2 / a} \sin (n \pi x / a), \quad n=1,2, \cdots .
$$

Lemma 4. (Huang [2]) If $f:[0, a] \rightarrow \mathbf{R}$ is a concave function, then

$$
\int_{0}^{a} f(x) \sin ^{2}\left(\frac{n \pi x}{a}\right) d x \geq \frac{1}{2} \int_{0}^{a} f(x) d x
$$

for $n=1,2, \cdots$.

With this lemma, we can now prove the following result for concave densities.

Theorem 5. If $\rho(x)$ is a concave density on $[0, a]$, then, for any $k \geq 1$,

$$
\sum_{n=1}^{k} \frac{1}{\lambda_{n}[\rho]} \geq\left(\frac{1}{a} \int_{0}^{a} \rho(x) d x\right) \sum_{n=1}^{k} \frac{1}{\lambda_{n}[1]}
$$


Moreover, the equality holds if and only if $\rho$ is constant.

Proof. To compare the eigenvalues $\lambda_{n}[\rho]$ with $\lambda_{n}[1]$, we consider the oneparameter family of densities: $\rho(x, t)=t \rho(x)+(1-t) \cdot 1$, where $0 \leq t \leq 1$. Then $\frac{\partial \rho}{\partial t}(x, t)=\rho(x)-1$ and $\frac{\partial^{2} \rho}{\partial t^{2}}(x, t)=0$. As in the proof of Theorem 3, we fix $k$ and let $\Lambda(t)=\sum_{n=1}^{k} \frac{1}{\lambda_{n}(t)}$. It then follows from Theorem 2 and (2) that

$$
\begin{aligned}
\sum_{n=1}^{k} \frac{1}{\lambda_{n}[\rho]}-\sum_{n=1}^{k} \frac{1}{\lambda_{n}[1]} & =\Lambda(1)-\Lambda(0) \\
& \geq \Lambda^{\prime}(0) \\
& =\sum_{n=1}^{k} \frac{1}{\lambda_{n}[1]} \int_{0}^{a} \frac{\partial \rho}{\partial t}(x, 0) u_{n}^{2}(x, 0) d x \\
& =\sum_{n=1}^{k} \frac{1}{\lambda_{n}[1]}\left\{\frac{2}{a} \int_{0}^{a} \rho(x) \sin ^{2}(n \pi x / a) d x-1\right\}
\end{aligned}
$$

where $u_{n}(x, 0)=\sqrt{2 / a} \sin (n \pi x / a), n=1,2, \cdots$, are the normalized eigenfunctions corresponding to the constant density $\rho(x, 0)=1$. Hence

$$
\begin{aligned}
\sum_{n=1}^{k} \frac{1}{\lambda_{n}[\rho]} & \geq \frac{2}{a} \sum_{n=1}^{k} \frac{1}{\lambda_{n}[1]} \int_{0}^{a} \rho(x) \sin ^{2}(n \pi x / a) d x \\
& \geq\left(\frac{1}{a} \int_{0}^{a} \rho(x) d x\right) \sum_{n=1}^{k} \frac{1}{\lambda_{n}[1]}
\end{aligned}
$$

on using Lemma 4. This proves (6).

Finally, we examine the case of equality. Since $\rho$ is concave, so is $\rho_{s}$. Hence, by Theorem 3 and (8), we have

$$
\begin{aligned}
\sum_{n=1}^{k} \frac{1}{\lambda_{n}[\rho]} & \geq \sum_{n=1}^{k} \frac{1}{\lambda_{n}\left[\rho_{s}\right]} \\
& \geq \frac{2}{a} \sum_{n=1}^{k} \frac{1}{\lambda_{n}[1]} \int_{0}^{a} \rho_{s}(x) \sin ^{2}(n \pi x / a) d x \\
& \geq\left(\frac{1}{a} \int_{0}^{a} \rho_{s}(x) d x\right) \sum_{n=1}^{k} \frac{1}{\lambda_{n}[1]} \\
& =\left(\frac{1}{a} \int_{0}^{a} \rho(x) d x\right) \sum_{n=1}^{k} \frac{1}{\lambda_{n}[1]} .
\end{aligned}
$$

Thus, by Theorem 3 and Lemma 4, equality holds in (6) only when $\rho=\rho_{s}$ and

$$
2 \int_{0}^{a} \rho_{s}(x) \sin ^{2}\left(\frac{n \pi x}{a}\right) d x=\int_{0}^{a} \rho_{s}(x) d x
$$


for all $n=1,2, \cdots, k$. To see that these conditions imply that $\rho$ is constant, we take $n=1$ and note that both $\rho_{s}$ and $\sin ^{2}(\pi x / a)$ are symmetric about $x=a / 2$ and are monotone increasing on $[0, a / 2]$. It follows that

$$
2 \int_{0}^{a} \rho_{s}(x) \sin ^{2}\left(\frac{\pi x}{a}\right) d x \geq \frac{2}{a} \int_{0}^{a} \rho_{s}(x) d x \int_{0}^{a} \sin ^{2}\left(\frac{\pi x}{a}\right) d x=\int_{0}^{a} \rho_{s}(x) d x .
$$

Moreover, the equality holds here only when $\rho_{s}$ is constant. This together with the condition $\rho=\rho_{s}$ completes the proof of the theorem.

The above comparison techniques also work in the case $\rho(x, t)=\rho(x)^{t}$.

Theorem 6. Let $\rho(x)$ be a density on $[0, a]$ such that $\log \rho(x)$ is concave. Then, for any $k \geq 1$,

$$
\sum_{n=1}^{k} \frac{1}{\lambda_{n}[\rho]} \geq\left(1+\frac{1}{a} \int_{0}^{a} \log \rho(x) d x\right) \sum_{n=1}^{k} \frac{1}{\lambda_{n}[1]} .
$$

Proof. The proof is similar to that of Theorem 5. Here we take $\rho(x, t)=\rho(x)^{t}$. Then $\frac{\partial \rho}{\partial t}(x, t)=\rho(x)^{t} \log \rho(x)$ and $\frac{\partial^{2} \rho}{\partial t^{2}}(x, t)=\rho(x)^{t}[\log \rho(x)]^{2} \geq 0$. Corresponding to (7), we obtain

$$
\begin{aligned}
\sum_{n=1}^{k} \frac{1}{\lambda_{n}[\rho]}-\sum_{n=1}^{k} \frac{1}{\lambda_{n}[1]} & \geq \sum_{n=1}^{k} \frac{1}{\lambda_{n}[1]} \int_{0}^{a} \frac{\partial \rho}{\partial t}(x, 0) u_{n}^{2}(x, 0) d x \\
& =\frac{2}{a} \sum_{n=1}^{k} \frac{1}{\lambda_{n}[1]} \int_{0}^{a}[\log \rho(x)] \sin ^{2}(n \pi x / a) d x
\end{aligned}
$$

Since $\log \rho(x)$ is concave, the theorem now follows from this and Lemma 4.

\section{APPENDIX}

In this appendix, we give an elementary proof of Theorem 1.

By the formula (2), we have

$$
\frac{-\lambda_{n}^{\prime}(t)}{\lambda_{n}(t)}=\int_{0}^{a} \frac{\partial \rho}{\partial t}(x, t) u_{n}^{2}(x, t) d x
$$

Differentiating (9) with respect to $t$ gives

$$
\begin{aligned}
\int_{0}^{a} & \frac{\partial^{2} \rho}{\partial t^{2}}(x, t) u_{n}^{2}(x, t) d x+2 \int_{0}^{a} \frac{\partial \rho}{\partial t}(x, t) u_{n}(x, t) \frac{\partial u_{n}}{\partial t}(x, t) d x \\
= & \frac{\left[\lambda_{n}^{\prime}(t)\right]^{2}-\lambda_{n}(t) \lambda_{n}^{\prime \prime}(t)}{\lambda_{n}^{2}(t)} \\
& =\left[\int_{0}^{a} \frac{\partial \rho}{\partial t}(x, t) u_{n}^{2}(x, t) d x\right]^{2}-\frac{\lambda_{n}^{\prime \prime}(t)}{\lambda_{n}(t)} .
\end{aligned}
$$


So, it suffices to show that

$$
\begin{aligned}
& 2 \int_{0}^{a} \frac{\partial \rho}{\partial t}(x, t) u_{n}(x, t) \frac{\partial u_{n}}{\partial t}(x, t) d x \\
& =-\left[\int_{0}^{a} \frac{\partial \rho}{\partial t}(x, t) u_{n}^{2}(x, t) d x\right]^{2} \\
& \quad-\sum_{j \neq n} \frac{2 \lambda_{n}(t)}{\lambda_{n}(t)-\lambda_{j}(t)}\left[\int_{0}^{a} \frac{\partial \rho}{\partial t}(x, t) u_{n}(x, t) u_{j}(x, t) d x\right]^{2} .
\end{aligned}
$$

Since $\left\{u_{j}(\cdot, t)\right\}_{j=1}^{\infty}$ forms a complete orthonormal basis for $L_{\rho(\cdot, t)}^{2}([0, a])$, we can write

$$
\frac{\partial u_{n}}{\partial t}(x, t)=\sum_{j=1}^{\infty}\left\{\int_{0}^{a} \frac{\partial u_{n}}{\partial t}(x, t) u_{j}(x, t) \rho(x, t) d x\right\} u_{j}(x, t)
$$

The normalization condition $\int_{0}^{a} u_{n}^{2}(x, t) \rho(x, t) d x=1$ implies that

$$
\int_{0}^{a} \frac{\partial u_{n}}{\partial t}(x, t) u_{n}(x, t) \rho(x, t) d x=-\frac{1}{2} \int_{0}^{a} u_{n}^{2}(x, t) \frac{\partial \rho}{\partial t}(x, t) d x .
$$

Now differentiating the equation

$$
u_{n}^{\prime \prime}(x, t)+\lambda_{n}(t) \rho(x, t) u_{n}(x, t)=0
$$

with respect to $t$ gives

$$
\begin{aligned}
& \frac{\partial u_{n}^{\prime \prime}}{\partial t}(x, t)+\lambda_{n}(t)\left[\rho(x, t) \frac{\partial u_{n}}{\partial t}(x, t)+\frac{\partial \rho}{\partial t}(x, t) u_{n}(x, t)\right] \\
& \quad=-\lambda_{n}^{\prime}(t) \rho(x, t) u_{n}(x, t) .
\end{aligned}
$$

Multiplying (13) by $u_{j}(x, t), j \neq n$, and then integrating over $[0, a]$, we obtain

$$
\begin{aligned}
& \int_{0}^{a} \frac{\partial u_{n}^{\prime \prime}}{\partial t}(x, t) u_{j}(x, t) d x+\lambda_{n}(t) \\
& \quad \int_{0}^{a}\left[\rho(x, t) \frac{\partial u_{n}}{\partial t}(x, t)+\frac{\partial \rho}{\partial t}(x, t) u_{n}(x, t)\right] u_{j}(x, t) d x \\
& =-\lambda_{n}^{\prime}(t) \int_{0}^{a} \rho(x, t) u_{n}(x, t) u_{j}(x, t) d x \\
& =0 .
\end{aligned}
$$


Using integration by parts twice and the boundary conditions $u_{n}(0, t)=u_{n}(a, t)=$ 0 , we find that

$$
\begin{aligned}
\int_{0}^{a} \frac{\partial u_{n}^{\prime \prime}}{\partial t}(x, t) u_{j}(x, t) d x & =\int_{0}^{a} \frac{\partial u_{n}}{\partial t}(x, t) u_{j}^{\prime \prime}(x, t) d x \\
& =-\lambda_{j}(t) \int_{0}^{a} \rho(x, t) \frac{\partial u_{n}}{\partial t}(x, t) u_{j}(x, t) d x
\end{aligned}
$$

This together with (14) gives, for $j \neq n$,

$$
\begin{aligned}
& \int_{0}^{a} \frac{\partial u_{n}}{\partial t}(x, t) u_{j}(x, t) \rho(x, t) d x \\
& \quad=\frac{\lambda_{n}(t)}{\lambda_{j}(t)-\lambda_{n}(t)} \int_{0}^{a} \frac{\partial \rho}{\partial t}(x, t) u_{n}(x, t) u_{j}(x, t) d x
\end{aligned}
$$

It follows from (11), (12) and (15) that

$$
\begin{aligned}
\frac{\partial u_{n}}{\partial t}(x, t)= & -\frac{1}{2}\left\{\int_{0}^{a} u_{n}^{2}(x, t) \frac{\partial \rho}{\partial t}(x, t) d x\right\} u_{n}(x, t) \\
& +\sum_{j \neq n} \frac{\lambda_{n}(t)}{\lambda_{j}(t)-\lambda_{n}(t)}\left\{\int_{0}^{a} \frac{\partial \rho}{\partial t}(x, t) u_{n}(x, t) u_{j}(x, t) d x\right\} u_{j}(x, t) .
\end{aligned}
$$

Substituting (16) into (10), we obtain the desired result. This completes the proof of Theorem 1.

\section{REFERENCES}

1. M.-J. Huang, On the eigenvalue ratio for vibrating strings, Proc. Amer. Math. Soc., 127 (1999), 1805-1813.

2. M.-J. Huang, Comparison theorems for eigenvalues of one-dimensional Schiodinger operators, J. Math. Kyoto Univ., 43 (2003), 465-474.

3. J. B. Keller, The minimum ratio of two eigenvalues, SIAM J. Appl. Math., 31 (1976), 485-491.

4. G. Pólya and M. Schiffer, Convexity of functionals by transplantation, J. Analyse Math., 3 (1954), 245-345.

\section{Min-Jei Huang}

Department of Mathematics,

National Tsing Hua University,

Hsinchu 30043, Taiwan.

E-mail: mjhuang@math.nthu.edu.tw 